\title{
Santé mentale des exilés en France : entre impuissance et créativité
}

Mental Health of Exiles in France: Between Powerlessness and Creativity Salud mental de los exiliados en Francia: entre impotencia y creatividad

Marie-Caroline Saglio-Yatzimirsky et Laure Wolmark

\section{(2) OpenEdition}

Édition électronique

URL : https://journals.openedition.org/remi/10558

DOI : 10.4000/remi.10558

ISSN : $1777-5418$

Éditeur

Université de Poitiers

Édition imprimée

Date de publication : 1 octobre 2018

Pagination : 21-27

ISBN : 979-10-90426-62-7

ISSN : 0765-0752

Référence électronique

Marie-Caroline Saglio-Yatzimirsky et Laure Wolmark, « Santé mentale des exilés en France : entre

impuissance et créativité », Revue européenne des migrations internationales [En ligne], vol. 34 - n² et 3

2018, mis en ligne le 28 décembre 2018, consulté le 14 avril 2022. URL : http://

journals.openedition.org/remi/10558; DOI : https://doi.org/10.4000/remi.10558 


\title{
Chronique d'actualité
}

\section{Santé mentale des exilés en France : entre impuissance et créativité}

\author{
Marie-Caroline Saglio-Yatzimirsky ${ }^{1}$ et \\ Laure Wolmark ${ }^{2}$
}

La récente loi asile et immigration publiée le 10 septembre 2018 a apporté son lot de mesures contradictoires qui limitent le droit des exilés. Parmi cellesci, certaines concernent leur santé, et en particulier leur santé mentale. La loi sur le droit des étrangers en France de mars 2016 avait déjà restreint le droit au séjour pour soin en transférant l'évaluation médicale des étrangers malades des médecins des Agences régionales de santé (ARS) dépendant du ministère de la Santé aux médecins de I'Office français de I'immigration et intégration (OFII), autrement dit au ministère de l'Intérieur ${ }^{3}$. La dernière loi empêche les déboutés du droit d'asile faisant l'objet d'une mesure d'éloignement de solliciter un tel titre de séjour pour soin, sauf éléments nouveaux. Implicitement, cela semble supposer que les demandes de déboutés étaient opportunistes plus que fondées sur un besoin réel de soins.

Dans le même sens, la loi asile et immigration exige donc désormais que le demandeur d'asile fasse conjointement la demande d'asile stricto sensu pour raisons politiques et la demande de séjour pour soin. Or, de nombreux exilés ne peuvent préciser leurs maladies et troubles qu'après recours à une prise en charge qui évalue leur état de santé et donc une fois effectif leur accès aux services de soins. Celui-ci est retardé par la situation souvent très confuse des primo-arrivants, mal informés des procédures administratives complexes, par leur difficulté d'obtention de la Protection universelle maladie (PUMA), remplaçant la Couverture maladie universelle (CMU), par I'absence d'interprétariat professionnel, ainsi que par l'instabilité de leur résidence rendant incertaine leur sectorisation.

\footnotetext{
1 Anthropologue, Psychologue clinicienne à I'Hôpital Avicenne, Inalco-CESSMA-ANR Liminal, Institut Convergences Migrations, Inalco 65 rue des grands moulins, 75013 Paris ; marie-caroline.yatzimirsky@inalco.fr

2 Psychologue clinicienne, coordinatrice nationale santé mentale au Comede (Comité pour la santé des exilés), 78 avenue de la Division du Général Leclerc, 94270 Le KremlinBicêtre ; laure.wolmark@comede.org

3 Pour un historique sur le droit au séjour pour soin des malades étrangers en France, et ses vicissitudes, voir Izambert (2011). Plus récemment, sur la loi de 2016, voir Math (2017).
} 
II n'est pas ici seulement question de procédures. La portée effective et symbolique de ces mesures exprime bien le déni, sinon le mépris, du soin pour les exilés et a fortiori du soin en santé mentale puisque près d'un quart des demandes de titre de séjour pour raison médicale relèvent de la santé mentale et en particulier du psychotraumatisme (OFII, 2018). La limitation du droit au séjour pour soin a des conséquences graves, car elle agit comme facteur de précarisation sociale et psychique des exilés, en même temps qu'elle déstabilise les soignants. Dans un climat de suspicion généralisée, les soignants des hôpitaux et des associations, psychiatres, psychologues qui accompagnent les exilés peuvent se sentir mis en doute, dans la mesure où leurs préconisations pour un traitement en France sont souvent contestées. Cette suspicion infuse aujourd'hui les pratiques cliniques, où l'on observe des limites de plus en plus sensibles dans la réception des exilés en même temps que d'importants manques de moyens.

Certes, la demande de soin en santé mentale, spécifique quand il s'agit de la population d'exilés, a toujours débordé les cadres de soin existant, car s'il est question de santé publique, il est aussi question d'accueil au sens politique et éthique. Par ailleurs, les exilés présentent des particularités, dont la langue et la culture "étrangères", qui complexifient les possibilités de réponses cliniques adéquates.

Ces réponses, a fortiori depuis ladite "crise de 2015 " qui a vu croître le nombre des primo-arrivants, sont aujourd'hui limitées par manque de moyens et de formation. Ainsi les exilés se font les révélateurs, à leur corps défendant, des failles et des faiblesses du système de soin public, et en particulier de la psychiatrie. De l'autre côté, la fragilisation des soignants s'est accentuée en même temps que la politisation croissante de la question, entrainant toujours plus de clivages et de malentendus sur le sujet de l'accès aux soins.

Toutefois, parce qu'elle déborde le cadre habituel de la psychiatrie, des institutions, des théorisations, la santé mentale des exilés force à l'invention et à la créativité. Elle renseigne aussi sur la place de la parole dans le soin : au-delà de la demande de preuves dans une ère du doute qui étouffe la subjectivité des exilés représentés tour à tour comme menteurs ou profiteurs comme le dénonçait récemment un collectif de soignants (Collectif, 2018), il existe aussi des espaces de soin où le sujet peut être entendu dans toutes ses dimensions.

\section{La complexité de la prise en charge en santé mentale des exilés}

En septembre 2018, un article de Laurent Chambaud et Fabienne Azzedine, de I'EHESP, cherchait à déconstruire dans La Tribune le préjugé qui associe santé des migrants et risque de contagion : "Première idée reçue : les migrants apporteraient des maladies en France" (Chambaud et Azzedine, 2018). Non seulement, rappellent les auteurs, l'état de santé des immigrés à leur arrivée est plutôt meilleur que celui des personnes nées en France, mais cet état a tendance à se dégrader avec la durée de leur séjour, comme le montre aussi l'étude Trajectoires et origines (TeO) de I'INED (Hamel et Moisy, 2016). De plus, si la santé physique des migrants est relativement bonne à leur arrivée, les troubles 
psychiques, particulièrement liés aux violences, sont plus fréquents. Les auteurs avancent la proportion d'une personne primo-arrivante sur cinq en situation de migration contrainte souffrant d'un trouble psychique.

Le centre de santé du Comede (Comité pour la santé des exilés), situé dans I'hôpital de Bicêtre, reçoit des personnes exilées majoritairement primo-arrivantes qui connaissent des difficultés d'accès aux soins. Parmi les causes de morbidité, $36 \%$ des diagnostics posés par les médecins du Comede concernent des troubles psychiques. Les syndromes psychotraumatiques - dont les traumas complexes liés à des agressions répétées (maltraitances, tortures, blessures, viols, humiliations, etc.) - , les syndromes dépressifs et les troubles anxieux sont les plus fréquemment rencontrés parmi les patients (respectivement $68 \%$, $22 \%$ et $8 \%$; voir Comede, 2017 : 78). Si ces troubles sont fortement corrélés aux antécédents de violence (Veisse et al., $2017: 408-409$ ), le lien avec la situation de précarité sociale en France apparaît aussi clairement (ibid. : 410).

Ainsi se dessinent les enjeux épidémiologiques et de santé publique de la santé mentale des migrants. L'introduction d'une récente synthèse bibliographie de I'IRDES les résume bien:

"L'étude de la santé des migrants est intéressante à plusieurs titres. En épidémiologie descriptive, elle relève d'une démarche d'investigation utilisée autrefois pour apprécier la part de l'environnement dans la genèse des maladies chroniques. En santé publique, elle entre dans le champ de l'examen des populations vulnérables. Mais il s'avère très difficile d'avoir une vision globale de l'état de santé des migrants, d'une part pour des raisons idéologiques, d'autre part du fait de la diversité des populations, des biais de déclaration dans les enquêtes ou de la sous-estimation des données de mortalité [...]. » (Safon, $2018: 3$ )

La rencontre clinique avec des personnes étrangères peut susciter désarroi et perplexité. Le désarroi de certains soignants est perceptible face à des patients dont les problématiques sont jugées trop complexes, parce que leur prise en charge relèverait d'une action globale, incluant le recours à l'interprétariat professionnel, dont une grande partie de la psychiatrie publique ne se donne pas les moyens. La perplexité s'exprime dans le sentiment que la santé mentale des exilés supposerait expertises et médiations spécifiques dans les domaines de la culture et du trauma, dont la plupart des soignants seraient dépourvus. L'exclusion et les obstacles à l'accès aux soins de santé mentale reposent ainsi sur l'idée d'une inadaptation mutuelle des exilés et des soignants. En cela, ils renvoient à une politique de l'accueil qui inclut, mais excède les seuls aspects des politiques de la santé.

En effet, que demandent les exilés aux soignants qui les reçoivent? Le désarroi et la perplexité que nous évoquions sont à comprendre aussi comme la réaction - consciente ou inconsciente - face à des demandes qui semblent toujours excéder la réponse que l'on peut leur donner : demandes d'attention, de présence, de soins pour être soulagé des cauchemars et de la peur, mais aussi demande d'aide dans la vie quotidienne, demandes de papiers, de certificats, demande de reconnaissance des violences, demande d'un lien humain, demande d'une place parmi les autres. Dans cet entremêlement des demandes, se donne à entendre la dimension psychique et subjective d'une politique défaillante de I'accueil. 


\section{Des réponses limitées}

La demande expresse de prise en charge de santé mentale des migrants s'est accentuée à partir de l'été 2015 avec l'arrivée de migrants en France directement liée à l'aggravation des situations politiques dans leur pays d'origine : populations d'Afghanistan, de Syrie, d'Irak ou d'Iran, de RD Congo, du Nigéria, du Soudan du Sud et Darfour, du Mali, de Guinée Conakry et de Côte d'Ivoire, des pays de la Corne de l'Afrique, etc. Ces populations, dont le nombre apparaît largement manipulé par le jeu politique et médiatique, représentent deux particularités : elles sont culturellement très diversifiées, accentuant la nécessité de recours à des interprètes dans les prises en charge; elles présentent des traumas complexes, car les trajectoires migratoires sont rendues particulièrement dangereuses et violentes (traversées de la Libye, politique de restriction et d'enfermement de certains pays côtiers pour limiter les départs, naufrages en Méditerranée, etc.).

Les politiques étatiques pour orienter les primo-arrivants sur le territoire français ne favorisent pas une réponse organisée et systématique. Ainsi, suite à la décision gouvernementale de démanteler ladite "jungle de Calais" en octobre 2016 et d'ouvrir des Centres d'accueil et d'orientation (CAO) sur tout le territoire national, vont s'exprimer des besoins importants en santé mentale dans des zones particulièrement démunies. Rappelons en effet que le "dispositif national d'accueil" qui comprend 80000 places d'hébergement en 2017 est conçu pour atteindre l'équilibre géographique et désengorger la région parisienne et les villes concentrant les arrivées des migrants. Les régions et les mairies accueillent donc divers CAO, CAES, PRADHA (dispositif réservé aux Dublinés), CHUM, plus récemment HUDA, etc., centres aux acronymes s'étoffant au rythme des spécificités administrativo-juridiques du traitement de l'asile, des exigences économiques ou de la problématique adoptée pour l'hébergement (par exemple, mixité ou non des espaces d'hébergement pour les migrants et d'autres populations dites "vulnérables", comme les SDF). La disparité de ce dispositif confronte nécessairement les primo-arrivants, mais aussi les services d'accueil, accompagnants, travailleurs sociaux, à une importante lacune de soins en santé mentale, soit que les services n'existent pas à proximité, soit que les soignants manquent de formation. La difficulté est renforcée par le nombre de "transferts" de demandeurs d'asile dans des structures d'hébergement, effectués quelquefois au mépris de leurs réseaux communautaires et amicaux. Non seulement les exilés sont fragilisés par les incohérences et les ruptures, mais aussi les accompagnants et les soignants.

Lors de la période d'arrivée qui est aussi celle de l'urgence, les primo-arrivants ne bénéficiant pas encore de la CMU sont pour beaucoup orientés vers les Permanences d'accès aux soins de santé (PASS) des hôpitaux, dont certaines offrent une consultation psychiatrique, même si cela reste très rare en dehors des grandes villes. Le recours (onéreux) à des services de traduction n'y est pas systématique. Beaucoup de médecins de PASS ne sont pas formés à recevoir des "patients traumas». La problématique est à la fois similaire et encore plus compliquée lorsqu'il s'agit d'organiser des suivis réguliers en santé mentale dans les Centres médico-psychologiques (CMP), pris dans la logique de la sectorisation. Le système sanitaire apparaît ainsi vite limité et saturé pour la population d'exilés. 
Une récente étude sur "l'état de santé d'une population vue au pôle santé du CPA ", Centre de Premier accueil de la Chapelle ouvert entre novembre 2016 et mars 2018 aux primo-arrivants ${ }^{4}$, évalue comme problématique centrale "les troubles psychiques graves notamment dus aux violences subies». Elle cite les problèmes posés par "une prise en charge retardée" alors que l'échantillon de patients enquêtés fait état de $61 \%$ de victimes de violences, dont $41 \%$ de violences psychiques. L'enquête conclut sur la nécessité d'améliorer la prise en charge psychologique dès le premier contact et de coordonner les soins avec les centres où sont transférés les exilés.

Pour mieux accéder à la demande d'une population très vulnérable, a fortiori lorsqu'elle est à la rue, d'autres propositions ont été développées, dont des maraudes avec présence de psychologues ou les EMPP (Équipe mobile psychiatrie précarité) des centres hospitaliers, qui s'adressent à la fois aux personnes en grande précarité et aux migrants. Les limites touchent alors à la difficulté de proposer une approche globale, qui tiendrait compte à la fois des besoins de soins en santé mentale et de l'accompagnement social et juridique. Les difficultés sont accrues lorsqu'il s'agit de familles, de femmes seules et de mineurs non accompagnés.

Ces limites des prises en charge en santé mentale ont plusieurs conséquences, tributaires du contexte : celles d'aggraver l'incompréhension des soignants et de la population civile face à un public d' "étrangers " perçus comme trop demandeurs sinon "profiteurs"; celle d'aggraver le sentiment d'impuissance des soignants puisque les lacunes de formation et le manque de moyens rencontrent ici la "la crise de la psychiatrie française» (Leboyer et Llorca, 2018). Enfin, à distinguer les réfugiés des autres exilés, demandeurs d'asile déboutés ou sans-papiers, on oublie un principe simple : pour une personne qui a été victime de traumas répétés et se révèle psychiquement extrêmement fragile, non seulement le soin est capital dès son arrivée, mais en plus l' «intégration" peut être compromise si les dispositifs d'accueil n'ont pas permis au sujet de renforcer ses ressources psychiques, son sentiment de sécurité, ses capacités de mise en mots.

\section{Des initiatives créatives}

Les limites et manques des services de soins en santé mentale, que renvoient les demandeurs d'asile, exigent paradoxalement des soignants des réponses particulièrement créatives. Ainsi d'autres relais se font jour, par exemple dans les initiatives associatives.

Bien avant la "crise de 2015 ", de nombreuses offres en soins psychiques ont tenté de répondre spécifiquement à la demande des exilés, couvrant un spectre large de prises en charge publiques et associatives, des consultations transculturelles à la clinique de l'exil, de groupes thérapeutiques (avec ou sans médiations) à des prises en charge d'inspiration cognitivo-comportementale, par des soignants allophones ou avec l'aide d'interprètes professionnels ou de

4 Présentation par les équipes du Samu social, hôpital Bichat et INSERM U1137 aux 19 èmes journées nationales d'infectiologie (Nantes, 13-15 juin 2018), [en ligne]. URL : http://www.infectiologie.com/UserFiles/File/jni/2018/com/jni2018-migrants-de-la-porte.pdf 
médiateurs. Ce paysage thérapeutique en santé mentale spécialement adressé aux "migrants" est certes bien plus important dans la capitale qu'en région.

Parallèlement, les créations d'espaces de réflexion et de centres de ressources se multiplient, en lien avec une clinique qui déborde les cadres existants. En effet, elle remet en question les positionnements et pratiques thérapeutiques habituelles, car il faut notamment arriver à lier l'approche psychiatrique du syndrome post-traumatique et une approche plus globale de la vulnérabilité propre à la migration.

Le champ de la santé mentale des migrants, s'il est un champ dans lequel les polémiques ont pu être vives (Fassin, 1999), demeure un lieu de vitalité théorique et clinique. II semble même que la situation d'urgence actuelle permette de renouveler l'espace des débats. En premier lieu, il est un champ de collaboration intense entre "le droit commun" et le milieu associatif, autour de l'orientation de certains patients, mais aussi de formations, de réunions institutionnelles ou d'échanges d'informations. D'autre part, la situation particulière des exilés primo-arrivants, leur position en marge de la cité, produit des transformations dans les modalités de prise en charge psychologique et psychiatrique. On peut citer ici, parmi de nombreuses autres initiatives, les maraudes des associations, mais aussi de collectifs non institutionnels de psychologues, les espaces d'écoute et de soin sous des tentes au sein des campements, les groupes de paroles ou d'échanges proposés pour des bénévoles et soutiens solidaires des exilés, les groupes thérapeutiques favorisant le lien social dans le cadre de partenariat "hors les murs" des associations. Ainsi, les dispositifs pour "aller vers" les exilés en souffrance se multiplient, et le "champ de la précarité " se révèle être un "laboratoire d'innovation sociale majeure " comme le rappelle Chambon (2018). Sur le plan théorique, la complexité et la perplexité face à l'intrication des dimensions médicales, sociales, juridiques, politiques et psychologiques conduisent à une hybridation théorique et clinique renouvelant les approches en incluant les apports des sciences sociales et politiques, de I'anthropologie, mais aussi de l'épidémiologie, de la santé publique, ou encore de la philosophie politique contemporaine dans la clinique auprès des exilés.

Un récent numéro de la Nouvelle Revue de Psychosociologie, intitulé "Migrants, réfugiés, exilés : résistance et créativités", rappelle dans son introduction qu'il y a "une double face de l'exil», ce qui signifie notamment "qu' [...] au pouvoir sur la vie répond toujours le pouvoir de la vie" (Lhuillier et Pestre, 2018 : 11). Aux mouvements d'exclusion des soins et de déni de la souffrance psychique des exilés répondent des espaces de créativité dans le soin guidés par des théories implicites ou explicites du "monde commun».

Dans les champs psychologiques et psychiatriques, c'est bien de la possibilité de l'écoute du sujet qu'il s'agit, que celui-ci se présente comme étranger, migrant, demandeur d'asile, exilé, etc. En demeurant à l'écoute de l'intime, la clinique singulière tente de répondre à une institution débordée par la violence du "réel " (Saglio-Yatzimirsky, 2018). Mais, face à l'ampleur des enjeux de santé publique liés à la santé mentale des exilés, cette clinique à l'écoute de la singularité doit pouvoir s'appuyer sur des réponses politiques ambitieuses et adaptées, visant à inclure les exilés au sein du système de soin, et plus généralement, de la société d'accueil. 


\section{Références bibliographiques}

Chambaud Laurent et Azzedine Fabienne (2018) Santé des migrants, les préjugés ont la vie dure, La Tribune, 07/09/2018, [en ligne]. URL : https://www.latribune.fr/ opinions/tribunes/sante-des-migrants-les-prejuges-ont-la-vie-dure-789150.html

Chambon Nicolas (Coord.) (2018) "Aller vers"... d'autres pratiques?, Rhizome, 68, 20 p., [en ligne] consulté le 02/11/2018. URL : http://www.ch-le-vinatier.fr/ orspere-samdarra/rhizome/anciens-numeros/rhizome-n68-aller-vers-d-autrespratiques/edito-2368.html

Collectif (2018) Demandeurs d'asile : «Partout autour de nous, nous observons la récurrence de la suspicion ", Le Monde, 04/04/2018, [en ligne]. URL : https://www. lemonde.fr/idees/article/2018/04/04/demandeurs-d-asile-partout-autour-de-nousnous-observons-la-recurrence-de-la-suspicion_5280564_3232.html

Comede (2017) Rapport d'activité et d'observation 2017, 120 p., [en ligne]. URL : http://www.comede.org/rapport-dactivite/

Fassin Didier (1999) L'ethnopsychiatrie et ses réseaux. L'influence qui grandit, Genèses. Sciences sociales et histoire, 35, pp. 146-171.

Hamel Christelle et Moisy Muriel (2016) Migrations et conditions de vie. Leur impact sur la santé, in Cris Beauchemin, Christelle Hamel et Patrick Simon Dirs., Trajectoire et origine. Enquête sur la diversité des populations en France, Paris, Ined Éditions, pp. 263-290.

Izambert Caroline (2011) Inhumain, mais pas trop, Vacarme, 54 (1), pp. 89-91, [en ligne]. URL : https://www.cairn.info/revue-vacarme-2011-1-page-89. htm?1=1\&Docld $=533074 \&$ hits $=6+5+$

Leboyer Marion et Llorca Pierre-Michel (2018) Psychiatrie : I'état d'urgence, Paris, Fayard, $432 \mathrm{p}$.

Lhuilier Dominique et Pestre Élise (2018) Introduction. La double face de I'exil, Nouvelle revue de psychosociologie, 1 (25), pp. 9-14.

Math Antoine (2017) Le droit au séjour pour raison médicale. La régularisation pour soin, Le Kremlin-Bicêtre, Comité pour la santé des exilés, 24 p., [en ligne]. URL : https://www.smg-pratiques.info/IMG/pdf/point_informatif_Idh_-_27_ janvier_-_etrangers_malades.pdf

OFII (2018) Rapport d'activité 2017, [en ligne]. URL : http://www.ofii.fr/lMG/pdf/ ofii_raa_2017.pdf

Safon Marie-Odile (2018) La santé des migrants. Bibliographie thématique, Paris, IRDES, [en ligne] consulté le 05/11/2018. URL : http://www.irdes.fr/documentation/syntheses/lasantedesmigrants.pdf

Saglio-Yatzimirsky Marie-Caroline (2018) La voix de ceux qui crient, rencontre avec des demandeurs d'asile, Paris, Albin Michel, 316 p.

Veisse Arnaud, Wolmark Laure, Revault Pascal, Giacopelli Maud, Bamberger Muriel et Zlatanova Zornitza (2017) Violences, vulnérabilité et troubles psychiques chez les migrants/exilés, BEH, 19-20, pp. 405-414. 